\title{
Redistribution of Kinetic Energy in Turbulent Flows
}

\author{
Alain Pumir, ${ }^{1,2,3,{ }^{*}}$ Haitao Xu, ${ }^{1,3, \dagger}$ Guido Boffetta, ${ }^{1,4}$ Gregory Falkovich, ${ }^{1,5,6}$ and Eberhard Bodenschatz ${ }^{1,3,7,8}$ \\ ${ }^{1}$ International Collaboration for Turbulence Research, D-37077, Göttingen, Germany \\ ${ }^{2}$ Ecole Normale Supérieure de Lyon and CNRS, F-69007 Lyon, France \\ ${ }^{3}$ Max-Planck Institute for Dynamics and Self-Organization (MPIDS), D-37077 Göttingen, Germany \\ ${ }^{4}$ Department of Physics and INFN, University of Torino, I-10125 Torino, Italy \\ ${ }^{5}$ Weizmann Institute of Science, Rehovot 76100, Israel \\ ${ }^{6}$ Institute for Information Transmission Problems, Moscow 127994, Russia \\ ${ }^{7}$ Institute for Nonlinear Dynamics, University of Göttingen, D-37077 Göttingen, Germany \\ ${ }^{8}$ Laboratory of Atomic and Solid State Physics and Sibley School of Mechanical \\ and Aerospace Engineering, Cornell University, Ithaca, New York 14853, USA
}

(Received 8 July 2014; published 13 October 2014)

\begin{abstract}
In statistically homogeneous turbulent flows, pressure forces provide the main mechanism to redistribute kinetic energy among fluid elements, without net contribution to the overall energy budget. This holds true in both two-dimensional (2D) and three-dimensional (3D) flows, which show fundamentally different physics. As we demonstrate here, pressure forces act on fluid elements very differently in these two cases. We find in numerical simulations that in 3D pressure forces strongly accelerate the fastest fluid elements, and that in 2D this effect is absent. In 3D turbulence, our findings put forward a mechanism for a possibly singular buildup of energy, and thus may shed new light on the smoothness problem of the solution of the Navier-Stokes equation in 3D.
\end{abstract}

DOI: $10.1103 /$ PhysRevX.4.041006

\section{INTRODUCTION}

Understanding the role of pressure is crucial in the study of fluid flows. This can be seen from the Navier-Stokes equation, which relates the acceleration of fluid particles to the various forces acting on them. In the case of incompressible fluids, it reads

$$
\frac{\partial \mathbf{u}}{\partial t}+\mathbf{u} \cdot \nabla \mathbf{u}=\mathbf{a}=-\nabla P+\mathbf{f}+\mathbf{D},
$$

where $\mathbf{u}(\mathbf{x}, t)$ is the fluid velocity, $\mathbf{a}$ is the fluid acceleration, and $P(\mathbf{x}, t)$ is the pressure. Without any loss of generality, the fluid density is set to 1 in Eq. (1). The terms $\mathbf{f}$ and $\mathbf{D}$ represent external body forces and dissipative processes acting on the fluid, respectively. In $3 \mathrm{D}, \mathbf{D}$ is due to the viscous forces only: $\mathbf{D}=\nu \nabla^{2} \mathbf{u}$, whereas in our 2D flow simulations in a box of finite size, the viscous dissipation is supplemented by a friction term $-\alpha \mathbf{u}$ to prevent accumulation of energy at the largest size in the system.

\footnotetext{
*alain.pumir@ens-lyon.fr

†haitao.xu@ds.mpg.de
}

Published by the American Physical Society under the terms of the Creative Commons Attribution 3.0 License. Further distribution of this work must maintain attribution to the author(s) and the published article's title, journal citation, and DOI.
Subject Areas: Fluid Dynamics, Nonlinear Dynamics, Statistical Physics

The role of pressure $P$ is to enforce incompressibility:

$$
\nabla \cdot \mathbf{u}=0
$$

Theoretical investigations of the role of pressure in turbulent flows [1-3] showed that the pressure gradient is the predominant term in Eq. (1) in determining the fluid acceleration. This prediction was supported by experimental measurements [4-7] and numerical simulations [8]. Consequently, there has been long-lasting effort to study the pressure alone $[9,10]$ and its role in determining the correlation functions of the velocity field [11-13].

Here, motivated by the recent study of the dynamics of the kinetic energy of fluid particles in turbulence and, in particular, by the observation of an asymmetric probability distribution function (PDF) of the instantaneous power of the forces acting on individual fluid particles [14,15], we investigate the contribution of pressure to the power $p=(d / d t)\left((1 / 2) \mathbf{u}^{2}\right)=\mathbf{u} \cdot \mathbf{a}$, which is the rate of change of the kinetic energy. Using Eq. (1), the power $p$ can be decomposed as

$$
p=\mathbf{u} \cdot \mathbf{a}=-\mathbf{u} \cdot \nabla P+\mathbf{u} \cdot \mathbf{f}+\mathbf{u} \cdot \mathbf{D} .
$$

In homogeneous flows, the pressure forces on average do no work and do not contribute to the balance of the kinetic energy, as follows from the exact relation [16-18], 


$$
\langle\mathbf{u} \cdot \nabla P\rangle=\langle\nabla \cdot(\mathbf{u} P)\rangle=\nabla \cdot\langle\mathbf{u} P\rangle=0,
$$

where \langle\rangle denotes an ensemble average over many flow realizations or, equivalently, a spatial average for homogeneous turbulence. In statistically stationary flows, in which $\langle p\rangle=\langle\mathbf{u} \cdot \mathbf{a}\rangle=(d / d t)\left\langle(1 / 2) \mathbf{u}^{2}\right\rangle=0$, the conservation of energy leads to $\langle\mathbf{u} \cdot \mathbf{f}\rangle=-\langle\mathbf{u} \cdot \mathbf{D}\rangle=\varepsilon$, where $\varepsilon$ is the turbulence energy dissipation rate.

Although the average of the pressure-gradient term $-\mathbf{u}$. $\nabla P$ is zero, its instantaneous values can be large. In this work, we study the statistics of the different terms in Eq. (3) using results from direct numerical simulations (DNS) in both $2 \mathrm{D}$ and $3 \mathrm{D}$. We show that the pressure contributions dominate the fluctuations of the instantaneous power in both $2 \mathrm{D}$ and 3D. Consistent with the well-known fact that turbulence dynamics depend in an essential way on the spatial dimension, we uncover important differences between the roles of the three terms in Eq. (3), in 2D and in 3D. In particular, our analysis demonstrates that the way pressure forces act on fast particles is very different in $2 \mathrm{D}$ and 3D. Namely, by conditioning $-\mathbf{u} \cdot \nabla P$ on the kinetic energy of fluid elements $(1 / 2) \mathbf{u}^{2}$, we observe that pressure does not lead to any energy transfer between fast and slow particles in 2D. In 3D, however, it on average slows down slow particles and accelerate fast ones: $\left\langle-\mathbf{u} \cdot \nabla P \mid \mathbf{u}^{2}\right\rangle$ is positive and grows with the energy even faster than $\mathbf{u}^{2}$ for $\mathbf{u}^{2} \gtrsim 2\left\langle\mathbf{u}^{2}\right\rangle$. Our observation of accelerating fast particles, which may suggest a runaway mechanism of the kinetic energy of particles in high Reynolds number flows, points to the importance of pressure forces in understanding fundamental properties of the Navier-Stokes equations in 3D [19-21]. Thus, our results concerning the redistribution of energy between fluid particles, implied by Eq. (4), may shed new light on the very different nature of the dynamics of turbulent flows in $2 \mathrm{D}$ and $3 \mathrm{D}$.

Our results are organized as follows. The analysis requires the accurate determination of pressure and velocity derivatives along particle trajectories, which at the present can be obtained only by DNS of turbulent flows. We briefly describe our numerical methods in Sec. II. In Sec. III, we discuss the magnitudes (variances) of the terms appearing in the decomposition equation [Eq. (3)] and show that the pressure term dominates. In Sec. IV, we analyze the third moments of the power in terms of the decomposition equatiion [Eq. (3)]. Finally, in Sec. V, we study and present evidence for a pressure-induced kinetic energy runaway mechanism in 3D.

\section{NUMERICAL METHODS}

The numerical results presented here are obtained by directly solving the equation of motion, Eqs. (1) and (2), in a periodic box of size $2 \pi$, using pseudospectral methods. In all cases, the flow is maintained stationary by using an external forcing term $\mathbf{f}$ in the Navier-Stokes equations.

\section{A. Three-dimensional simulations}

In 3D, the dissipation term in Eq. (1) is due to viscosity only: $\mathbf{D}=\nu \nabla^{2} \mathbf{u}$. The forcing term is chosen in such a way that energy is injected at a large scale $l_{F}$, comparable to the size of the system. The energy injected at that given scale cascades to smaller scales to be dissipated [22]. The cascade proceeds with a constant energy flux $\varepsilon$ down to the smallest scale $\eta=\left(\nu^{3} / \varepsilon\right)^{1 / 4}$, the Kolmogorov scale. The Reynolds number of the flow is a quantitative representation of the ratio between the length scales $l_{F} / \eta$. In most studies of turbulence, the Taylor microscale Reynolds number is used, defined as $R_{\lambda}=\left\langle u_{x}^{2}\right\rangle^{1 / 2} \lambda / \nu$, where $u_{x}$ is the velocity component in the $x$ direction and $\lambda$ is the Taylor microscale: $\lambda \equiv\left[\left\langle u_{x}^{2}\right\rangle /\left\langle\left(\partial_{x} u_{x}\right)^{2}\right\rangle\right]^{1 / 2}$. With this definition, ignoring possible intermittency corrections, the ratio $l_{F} / \eta$ and $R_{\lambda}$ are related by

$$
R_{\lambda} \propto\left(l_{F} / \eta\right)^{2 / 3} .
$$

The data reported here cover the range of Reynolds numbers $115 \leq R_{\lambda} \leq 430$. The lower Reynolds number runs (with $192^{3}$ or $384^{3}$ spectral modes) are carried out on local workstations at ENS Lyon. The flows are forced by keeping a fixed energy in the low wave number modes $(|k|<1.5)$, which evolves according to the truncated Euler equations [23]. The resulting flow has been shown to be statistically homogeneous and isotropic. By maintaining an adequate numerical resolution (the product $k_{\max } \times \eta$ is larger than 1.4 , where $k_{\max }$ is the largest mode faithfully simulated), the Reynolds numbers we reach in the calculations are $R_{\lambda}=115$ (with $192^{3}$ modes) and $R_{\lambda}=170$ (with $384^{3}$ modes). In both cases, the total number of samples to determine the statistics is larger than $10^{8}$.

The data at higher Reynolds number $\left(R_{\lambda}=430\right)$ are obtained from the Johns Hopkins Turbulence Database [24]. From the database, we extract the values of $\mathbf{u}, \mathbf{f}$, $\mathbf{D}=\nu \nabla^{2} \mathbf{u}$ and $\nabla P$ over a set of $128 \times 256 \times 512$ spatial points, at 16 instants of time, equally distributed over one large eddy turnover time. This results in $2.7 \times 10^{8}$ data points in the statistics.

\section{B. Two-dimensional simulations}

In 2D, the dissipation term in Eq. (1) involves, in addition to the viscous forces, a linear friction term: $\mathbf{D}=\nu \nabla^{2} \mathbf{u}-\alpha \mathbf{u}$. The forcing term $\mathbf{f}$ supplies energy and enstrophy into the fluid, at rates $\varepsilon_{I}$ and $\zeta_{I}$, respectively. Their magnitudes are determined by the forcing scale $l_{F}$ as $\left(\varepsilon_{I} / \zeta_{I}\right)^{1 / 2}=l_{F}$.

A specific property of $2 \mathrm{D}$ turbulence is the inverse energy cascade [25]. In the asymptotic limit of vanishingly small values of $\alpha$ and $\nu$, all of the energy cascades to large scales and the enstrophy cascades to small scales. At finite values of $\nu$ and $\alpha$, part of the energy flows to large scales, with a flux $\varepsilon$, and part of the energy flows down scale, with 
a flux $\varepsilon_{\nu}$. Similarly, part of the enstrophy goes to smaller scales (with a flux $\zeta_{\nu}$ ), while another part flows up scale (flux $\zeta$ ). Stationarity requires that $\varepsilon_{I}=\varepsilon_{\nu}+\varepsilon$ and $\zeta_{I}=\zeta_{\nu}+\zeta$. A simple balance of energy and enstrophy fluxes gives $\varepsilon_{\nu} / \varepsilon \propto \nu$ [26], which is indeed observed in numerical simulations [27]. A similar relation can be obtained for enstrophy fluxes.

At finite values of $\alpha$, the inverse energy cascade proceeds up to the scale $l_{\alpha} \approx \alpha^{-3 / 2} \varepsilon^{1 / 2}$, where friction dominates. Conversely, the direct enstrophy cascade proceeds down to the viscous scale $l_{\nu}$, given by $l_{\nu} \approx \nu^{1 / 2} \zeta_{\nu}^{-1 / 6}$.

In order to compare $2 \mathrm{D}$ and $3 \mathrm{D}$ simulations, it is convenient to introduce an equivalent of the Taylor microscale Reynolds number for the inverse energy cascade, based on the range of scales of the inverse cascade, by analogy with Eq. (5):

$$
R_{\alpha} \equiv\left(l_{\alpha} / l_{F}\right)^{2 / 3}
$$

Since we are interested here in a flow developing an inverse energy cascade, the time scale corresponding to the forcing length is much smaller than that at the size of the largest eddies. In this work, we choose to use a forcing that is white in time (formally zero correlation time). Other forcing schemes have been used in the literature [28-30]. Although some properties of the flow may change slightly, the precise choice of the forcing is not expected to affect the physics of the inverse cascade [15].

In this work, we present simulation results with Reynolds number in the range $26 \leq R_{\alpha} \leq 102$ obtained from DNS with up to $4096^{2}$ grid points.

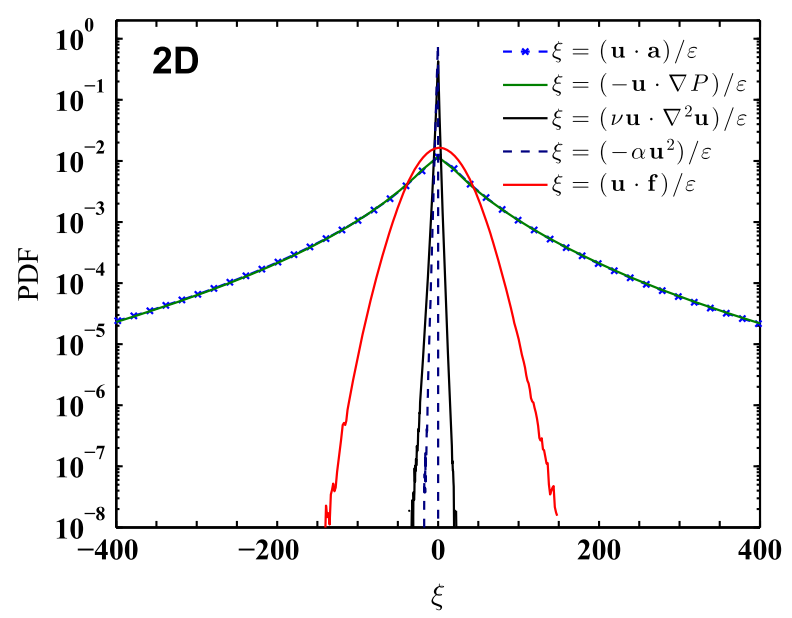

(a)

\section{CONTRIBUTIONS OF PRESSURE, DISSIPATION, AND FORCING TO THE VARIANCE OF POWER}

In Figs. 1(a) and 1(b), we show the PDFs of the instantaneous power $p$ and its various components in 2D and $3 \mathrm{D}$, respectively, including the contributions from the pressure-gradient term $-\mathbf{u} \cdot \nabla P$, the forcing term $\mathbf{u} \cdot \mathbf{f}$, the viscous dissipation term $\nu \mathbf{u} \cdot \nabla^{2} \mathbf{u}$, and the friction term $-\alpha \mathbf{u}^{2}$ in the 2D case, all made dimensionless by the turbulence energy flux in the cascade range $\varepsilon$. Judging from the extent of the tails of the PDFs, the fluctuations of the pressure term greatly exceed the fluctuations of the dissipation term and of the forcing term. In fact, for 3D turbulence, the following inequalities are observed to hold:

$$
\left\langle(\mathbf{u} \cdot \mathbf{f})^{2}\right\rangle \ll\left\langle\left(\nu \mathbf{u} \cdot \nabla^{2} \mathbf{u}\right)^{2}\right\rangle \ll\left\langle(\mathbf{u} \cdot \nabla P)^{2}\right\rangle
$$

Note that for 2D turbulence, the forcing varies on short times. Our choice of using a white-in-time forcing makes the interpretation of the instantaneous power subtle [15]. The $\mathbf{u} \cdot \mathbf{f}$ term is evaluated by averaging $\mathbf{u} \cdot \mathbf{f}$ over a time interval $\tau \lesssim \tau_{F}$, where $\tau_{F} \equiv\left(l_{F}^{2} / \varepsilon\right)^{1 / 3}$ is the characteristic turbulence cascade time scale at the forcing length scale. The variance of the forcing term, averaged over $\tau_{F}$, as can be seen from Fig. 1(a), is in fact larger than the variance of the friction term $-\alpha \mathbf{u}^{2}$. This can be qualitatively understood by noticing that in 2D the flow is forced at scales that are smaller than those at which friction acts, and as a result, the fluctuations in the forcing are larger than those of the friction term [31].

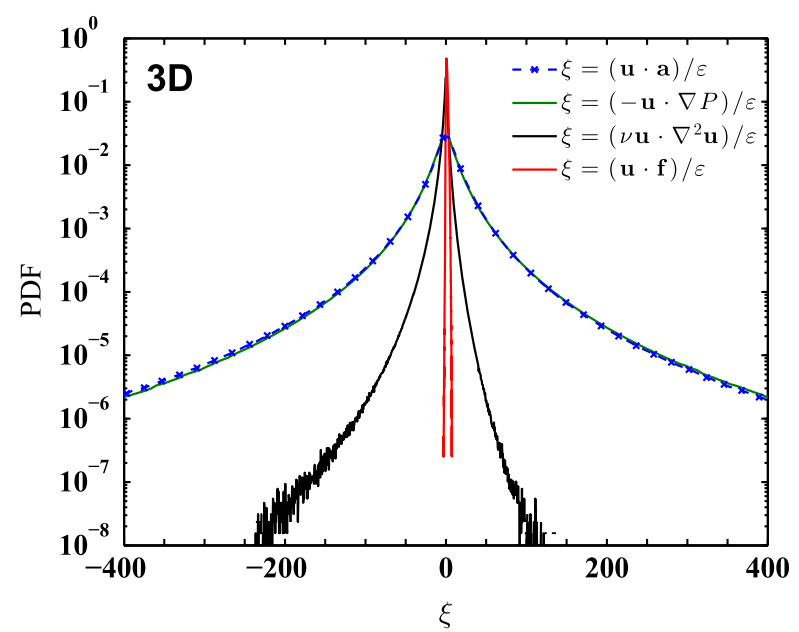

(b)

FIG. 1. Probability density functions (PDFs) of the instantaneous power $p=\mathbf{u} \cdot \mathbf{a}$ and its components in both 2D and 3D turbulence, all nondimensionalized with the turbulence energy flux $\varepsilon$. (a) The PDFs of $p / \varepsilon,-\mathbf{u} \cdot \nabla P / \varepsilon, \nu \mathbf{u} \cdot \nabla^{2} \mathbf{u} / \varepsilon$, $\mathbf{u} \cdot \mathbf{f} / \varepsilon$, and the friction term $-\alpha \mathbf{u}^{2} / \varepsilon$ from direct numerical simulations (DNS) of $2 \mathrm{D}$ turbulence at $R_{\alpha}=51$. (b) The PDFs of $p / \varepsilon$, the pressure-gradient term $-\mathbf{u} \cdot \nabla P / \varepsilon$, the viscous dissipation term $\nu \mathbf{u} \cdot \nabla^{2} \mathbf{u} / \varepsilon$, and the forcing term $\mathbf{u} \cdot \mathbf{f} / \varepsilon$ from 3D DNS at $R_{\lambda}=430$. In both $2 \mathrm{D}$ and 3D, the magnitudes of the instantaneous power and the contribution from the pressure gradient are much larger than those from the dissipation term and the external forcing. 


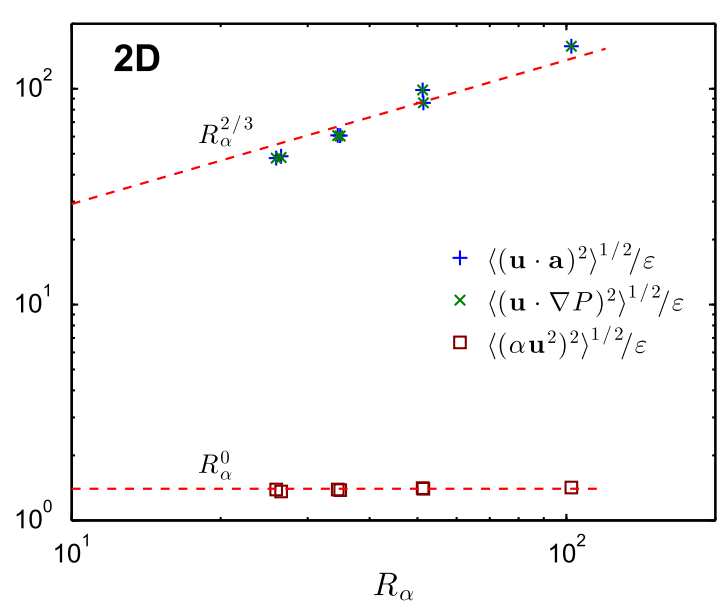

(a)

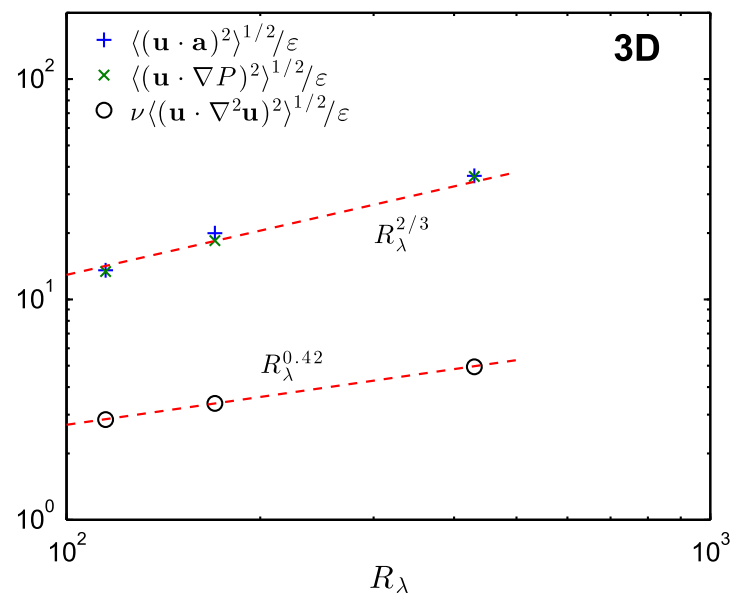

(b)

FIG. 2. Variation of the standard deviations of $\mathbf{u} \cdot \mathbf{a} / \varepsilon$ (blue pluses), $\mathbf{u} \cdot \nabla P / \varepsilon$ (green crosses), $\nu \mathbf{u} \cdot \nabla^{2} \mathbf{u} / \varepsilon$ (black circles), and $\alpha u^{2} / \varepsilon$ (brown squares) as functions of Reynolds numbers in both (a) $2 \mathrm{D}$ and (b) $3 \mathrm{D}$.

The Reynolds number dependence of the magnitudes of the various terms in Eq. (3) is shown in Figs. 2(a) and 2(b) for 2D and 3D turbulence, respectively.

As seen from Fig. 2(a), the standard deviation of $\mathbf{a} \cdot \mathbf{u}$ grows roughly as $R_{\alpha}^{2 / 3}$ [15], and is nearly indistinguishable from that of the pressure term $-\mathbf{u} \cdot \nabla P$, which overwhelmingly dominates any of the other terms in Eq. (3). The standard deviation of the frictional dissipation term $-\alpha \mathbf{u}^{2}$ is essentially constant over the entire range of Reynolds numbers studied. On the other hand, the standard deviation of the viscous dissipation term $\nu \mathbf{u} \cdot \nabla^{2} \mathbf{u}$ depends on the viscosity of the flow, consistent with the relation $\varepsilon_{\nu} / \varepsilon \propto \nu$, and thus, depends explicitly on the two parameters $\alpha$ and $\nu$, resulting in a more complicated dependence on the Reynolds number $R_{\alpha}$.

Similar conclusions apply in the 3D case; see Fig. 2(b). The fluctuations of $\mathbf{u} \cdot \mathbf{a},-\mathbf{u} \cdot \nabla P$, and $\nu \mathbf{u} \cdot \nabla^{2} \mathbf{u}$ all grow plausibly as power laws of $R_{\lambda}$, at least over the range of values covered in this study. More specifically, the fluctuation of $\mathbf{u} \cdot \mathbf{a}$ is dominated by the pressure term $-\mathbf{u} \cdot \nabla P$, and the standard deviations of both terms grow approximately as $R_{\lambda}^{2 / 3}$ [15]. In comparison, the standard deviation of the dissipation term, $\nu \mathbf{u} \cdot \nabla^{2} \mathbf{u}$, grows approximately as $R_{\lambda}^{0.42}$. As a consequence, the direct contribution to the power from viscous dissipation, as measured by the second moment, becomes weaker when the Reynolds number increases. We note that this is consistent with the results of Vedula and Yeung [8], who observed a similar trend for the individual variances of $\nabla P$ and $\nu \nabla^{2} \mathbf{u}$ while studying the contributions to the acceleration variance using Eq. (1).

One may attempt to evaluate the pressure contribution to power by assuming $\left\langle(\mathbf{u} \cdot \nabla P)^{2}\right\rangle \approx\left\langle\mathbf{u}^{2}\right\rangle\left\langle(\nabla P)^{2}\right\rangle$ and then using the standard estimate $|\nabla P| \approx|\mathbf{a}| \propto\left(\varepsilon^{3} / \nu\right)^{1 / 4}$ to obtain $\left\langle(\mathbf{u} \cdot \nabla P)^{2}\right\rangle / \varepsilon^{2} \propto R_{\lambda}$ or $R_{\alpha}$. The observed growth, $\left\langle(\mathbf{u} \cdot \nabla P)^{2}\right\rangle / \varepsilon^{2} \propto R_{\lambda}^{4 / 3}$ or $R_{\alpha}^{4 / 3}$, is faster than that suggested by the above simple argument, even taking into account the intermittency effects on the magnitudes of a or $\nabla P[32,33]$. This, therefore, implies that the correlations between $\mathbf{u}$ and $\nabla P$, both in terms of magnitude and alignment, depend on the Reynolds number.

\section{CONTRIBUTIONS OF PRESSURE, DISSIPATION, AND FORCING TO THE THIRD MOMENT OF POWER}

In view of the results of the previous section, it is natural to ask whether the third moment of power $p$ is also dominated by pressure forces. This question arises, in particular, in relation to our recent observation that the distributions of $p$ are negatively skewed in both 2D and 3D $[14,15]$. Quantifying the relative contributions of the various terms in Eq. (3) provides insight into the different physical effects involved in turbulent flows. The results summarized in this section indicate that the contributions of the various forces to the third moment depend crucially on the spatial dimension. More precisely, our results show that the third moment of the pressure term, $\left\langle(-\mathbf{u} \cdot \nabla P)^{3}\right\rangle$, contributes significantly to the third moment of power in $2 \mathrm{D}$, but plays a negligible role in $3 \mathrm{D}$.

\section{A. 2D flows}

We first discuss 2D turbulence, based on the moments recorded in Table I. More detailed information can be found in Appendix A.

The standard deviations of $p,-\mathbf{u} \cdot \nabla P$, and the dissipation terms due to viscosity $\nu \mathbf{u} \cdot \nabla^{2} \mathbf{u}$ and to friction $-\alpha \mathbf{u}^{2}$, shown in row $M_{2}^{2 \mathrm{D}}$ of Table I for 2D turbulent flows, indicate that $-\mathbf{u} \cdot \nabla P$ dominates the fluctuations of $p$, in agreement with the conclusion of Sec. III. The flatness of 
$-\mathbf{u} \cdot \nabla P$ is also significantly larger than the flatness of the dissipative terms, $\nu \mathbf{u} \cdot \nabla^{2} \mathbf{u}$ and $-\alpha \mathbf{u}^{2}$ (row $F l^{2 \mathrm{D}}$ of Table I). Interestingly, the flatness of $-\mathbf{u} \cdot \nabla P$ grows by almost a factor of 2 when $R_{\alpha}$ increases from 26 to 102 . The observation that the fourth moment of $-\mathbf{u} \cdot \nabla P$ grows faster than $\left.\langle(-\mathbf{u} \cdot \nabla) P)^{2}\right\rangle$ is unexpected. It means that the tails of the distribution of the pressure contribution become more extended when the Reynolds number increases. This should be contrasted with the well-established result that Eulerian velocity differences in 2D turbulent flows do not reveal any trace of intermittency [26].

The contribution to the third moment of $p$ from the pressure gradient $\left\langle(-\mathbf{u} \cdot \nabla P)^{3}\right\rangle$ is the largest term in $2 \mathrm{D}$, providing by itself $\sim 60 \%$ of $\left\langle p^{3}\right\rangle$. In fact, as shown in row $M_{3}^{2 \mathrm{D}}$ of Table I, the two largest contributions, the third moment of the pressure term and the correlation between the pressure gradient and the friction force, nearly account for the entire $\left\langle p^{3}\right\rangle$ :

$$
\left\langle p^{3}\right\rangle \approx\left\langle(-\mathbf{u} \cdot \nabla P)^{3}\right\rangle+\left\langle 3(-\mathbf{u} \cdot \nabla P)^{2}\left(-\alpha \mathbf{u}^{2}\right)\right\rangle
$$

We note that in row $M_{3}^{2 D}$ of Table I the contributions from the pressure-forcing-term correlation, $\left\langle(-\mathbf{u} \cdot \nabla P)^{2}(\mathbf{u} \cdot \mathbf{f})\right\rangle$, and the pressure-viscous-term correlation, $\left\langle(-\mathbf{u} \cdot \nabla P)^{2}\left(\nu \mathbf{u} \cdot \nabla^{2} \mathbf{u}\right)\right\rangle$, almost cancel each other completely. The direct contributions from the dissipation terms, $\left\langle\left(\nu \mathbf{u} \cdot \nabla^{2} \mathbf{u}\right)^{3}\right\rangle$ and $\left\langle\left(-\alpha \mathbf{u}^{2}\right)^{3}\right\rangle$, are all negligible due to the small variances of these terms.

Given the very large contribution of $-\mathbf{u} \cdot \nabla P$ to the variance of $p$ (Fig. 1), one may have surmised that the third moment of the pressure gradient should also significantly contribute to $\left\langle p^{3}\right\rangle$. The study of the 3D case in Sec. IV B shows, however, that this assumption is, in general, not correct.

\section{B. 3D flows}

We now discuss 3D turbulence, based on Table II. Further information can be found in Appendix B.

In agreement with the conclusion of Sec. III, the data shown in row $M_{2}^{3 \mathrm{D}}$ of Table II demonstrate that, in terms of second moments, the pressure term $-\mathbf{u} \cdot \nabla P$ dominates the fluctuations of $p$. The flatness of $-(\mathbf{u} \cdot \nabla P)$, indicated in row $F l^{3 \mathrm{D}}$ of Table II, grows from about 28 at $R_{\lambda}=115$ to a value close to 100 at $R_{\lambda}=430$. In comparison, the flatness of $\nu \mathbf{u} \cdot \nabla^{2} \mathbf{u}$ is significantly smaller, and does not increases as much with $R_{\lambda}$ : its value grows from about 30 at $R_{\lambda}=115$ to 56 at $R_{\lambda}=430$. The observed increase in flatness of the quantities shown in row $F l^{3 \mathrm{D}}$ is generally consistent with the well-known intermittency, characteristic

TABLE I. Second and higher moments of terms in Eq. (3) in 2D turbulence. Parameters in the simulation: $\alpha$ is the friction factor, $\nu$ is the viscousity, and $\varepsilon$ is the flux of energy in the inverse cacade. Row $M_{2}^{2 D}$ : Second moments of $p,-\mathbf{u} \cdot \nabla P, \nu \mathbf{u} \cdot \nabla^{2} \mathbf{u}$, and $-\alpha \mathbf{u}^{2}$, all made dimensionless by the energy flux $\varepsilon$. Row $F l^{2 \mathrm{D}}$ : Flatnesses of $-\mathbf{u} \cdot \nabla P, \nu \mathbf{u} \cdot \nabla^{2} \mathbf{u}$, and $-\alpha \mathbf{u}^{2}$. Row $S k^{2 \mathrm{D}}$ : Skewnesses of $p$, $-\mathbf{u} \cdot \nabla P, \nu \mathbf{u} \cdot \nabla^{2} \mathbf{u}$, and $-\alpha \mathbf{u}^{2}$. Row $M_{3}^{2 D}$ : Contributions to the third moment $\left\langle p^{3}\right\rangle$ from the pressure term, the viscous term, the friction term, and the three leading cross-correlation terms.

\begin{tabular}{|c|c|c|c|c|}
\hline & $R_{\alpha}$ & 26 & 51 & 102 \\
\hline & $\begin{array}{l}\alpha \\
\nu \\
\varepsilon\end{array}$ & $\begin{array}{c}0.08 \\
2.3 \times 10^{-6} \\
1.34 \times 10^{-3}\end{array}$ & $\begin{array}{c}0.04 \\
2.3 \times 10^{-6} \\
1.30 \times 10^{-3}\end{array}$ & $\begin{array}{c}0.02 \\
2.3 \times 10^{-6} \\
1.28 \times 10^{-3}\end{array}$ \\
\hline$M_{2}^{2 \mathrm{D}}$ & $\begin{array}{c}\left\langle p^{2}\right\rangle / \varepsilon^{2} \\
\left\langle(-\mathbf{u} \cdot \nabla P)^{2}\right\rangle / \varepsilon^{2} \\
\left\langle\left(\left(\mathbf{u} \cdot \nabla^{2} \mathbf{u}\right)^{2}\right\rangle / \varepsilon^{2}\right. \\
\left\langle\left(-\alpha \mathbf{u}^{2}\right)^{2}\right\rangle / \varepsilon^{2}\end{array}$ & $\begin{array}{l}2.28 \times 10^{3} \\
2.28 \times 10^{3} \\
1.56 \\
1.93\end{array}$ & $\begin{array}{c}7.46 \times 10^{3} \\
7.40 \times 10^{3} \\
3.10 \\
1.96\end{array}$ & $\begin{array}{l}2.45 \times 10^{4} \\
2.48 \times 10^{4} \\
6.15 \\
2.02\end{array}$ \\
\hline$F l^{2 \mathrm{D}}$ & $\begin{array}{c}\left\langle(-\mathbf{u} \cdot \nabla P)^{4}\right\rangle /\left\langle(-\mathbf{u} \cdot \nabla P)^{2}\right\rangle^{2} \\
\left\langle\left(\nu \mathbf{u} \cdot \nabla^{2} \mathbf{u}\right)^{4}\right\rangle /\left\langle\left(\nu \mathbf{u} \cdot \nabla^{2} \mathbf{u}\right)^{2}\right\rangle^{2} \\
\left\langle\left(\left(-\alpha \mathbf{u}^{2}\right)^{4}\right\rangle /\left\langle\left(-\alpha \mathbf{u}^{2}\right)^{2}\right\rangle^{2}\right.\end{array}$ & $\begin{array}{l}17.1 \\
9.43 \\
5.49\end{array}$ & $\begin{array}{l}22.7 \\
8.82 \\
5.60\end{array}$ & $\begin{array}{l}31.5 \\
8.56 \\
6.17\end{array}$ \\
\hline$S k^{2 \mathrm{D}}$ & $\begin{array}{c}\left\langle p^{3}\right\rangle /\left\langle p^{2}\right\rangle^{3 / 2} \\
\left\langle(-\mathbf{u} \cdot \nabla P)^{3}\right\rangle /\left\langle(-\mathbf{u} \cdot \nabla P)^{2}\right\rangle^{3 / 2} \\
\left\langle\left(\nu \mathbf{u} \cdot \nabla^{2} \mathbf{u}\right)^{3}\right\rangle /\left\langle\left(\nu \mathbf{u} \cdot \nabla^{2} \mathbf{u}\right)^{2}\right\rangle^{3 / 2} \\
\left\langle\left(-\alpha \mathbf{u}^{2}\right)^{3}\right\rangle /\left\langle\left(-\alpha \mathbf{u}^{2}\right)^{2}\right\rangle^{3 / 2}\end{array}$ & $\begin{array}{l}-0.28 \\
-0.13 \\
-1.99 \\
-2.04\end{array}$ & $\begin{array}{l}-0.20 \\
-0.11 \\
-1.60 \\
-2.06\end{array}$ & $\begin{array}{l}-0.12 \\
-0.068 \\
-1.23 \\
-2.14\end{array}$ \\
\hline$M_{3}^{2 \mathrm{D}}$ & $\begin{array}{c}\left\langle(-\mathbf{u} \cdot \nabla P)^{3}\right\rangle /\left\langle p^{3}\right\rangle \\
\left\langle\left(\nu \mathbf{u} \cdot \nabla^{2} \mathbf{u}\right)^{3}\right\rangle /\left\langle p^{3}\right\rangle \\
\left\langle\left(-\alpha \mathbf{u}^{2}\right)^{3}\right\rangle /\left\langle p^{3}\right\rangle \\
3\left\langle(-\mathbf{u} \cdot \nabla P)^{2}\left(-\alpha \mathbf{u}^{2}\right)\right\rangle /\left\langle p^{3}\right\rangle \\
3\left\langle(-\mathbf{u} \cdot \nabla P)^{2}(\mathbf{u} \cdot \mathbf{f})\right\rangle /\left\langle p^{3}\right\rangle \\
3\left\langle(-\mathbf{u} \cdot \nabla P)^{2}\left(\nu \mathbf{u} \cdot \nabla^{2} \mathbf{u}\right)\right\rangle /\left\langle p^{3}\right\rangle\end{array}$ & $\begin{array}{c}0.47 \\
1.27 \times 10^{-4} \\
1.79 \times 10^{-4} \\
0.55 \\
-0.31 \\
0.29\end{array}$ & $\begin{array}{c}0.56 \\
6.78 \times 10^{-5} \\
4.39 \times 10^{-5} \\
0.42 \\
-0.25 \\
0.27\end{array}$ & $\begin{array}{c}0.56 \\
4.08 \times 10^{-5} \\
1.34 \times 10^{-5} \\
0.39 \\
-0.23 \\
0.27\end{array}$ \\
\hline
\end{tabular}


TABLE II. Second and higher moments of terms in Eq. (3) in 3D turbulence. Row $M_{2}^{3 \mathrm{D}}:$ Second moments of $p,-\mathbf{u} \cdot \nabla P$, and $\nu \mathbf{u} \cdot \nabla^{2} \mathbf{u}$, all made dimensionless by the energy flux $\varepsilon$. Row $F l^{3 \mathrm{D}}$ : Flatnesses of $-\mathbf{u} \cdot \nabla P$, and $\nu \mathbf{u} \cdot \nabla^{2} \mathbf{u}$. Row $S k^{3 \mathrm{D}}:$ Skewnesses of $p$, $-\mathbf{u} \cdot \nabla P$, and $\nu \mathbf{u} \cdot \nabla^{2} \mathbf{u}$. Row $M_{3}^{3 \mathrm{D}}$ : Contributions to the third moment $\left\langle p^{3}\right\rangle$ from the pressure term, the viscous term, and the two leading cross-correlation terms. The symbol * means that the terms are not directly available because of the implicit forcing scheme used in those simulations.

\begin{tabular}{|c|c|c|c|c|}
\hline & $R_{\lambda}$ & 115 & 170 & 430 \\
\hline$M_{2}^{3 \mathrm{D}}$ & $\begin{array}{c}\left\langle p^{2}\right\rangle / \varepsilon^{2} \\
\left\langle(-\mathbf{u} \cdot \nabla P)^{2}\right\rangle / \varepsilon^{2} \\
\left\langle\left(\nu \mathbf{u} \cdot \nabla^{2} \mathbf{u}\right)^{2}\right\rangle / \varepsilon^{2}\end{array}$ & $\begin{array}{c}1.84 \times 10^{2} \\
1.79 \times 10^{2} \\
8.12\end{array}$ & $\begin{array}{c}4.00 \times 10^{2} \\
3.42 \times 10^{2} \\
11.4\end{array}$ & $\begin{array}{c}1.32 \times 10^{3} \\
1.31 \times 10^{3} \\
24.5\end{array}$ \\
\hline$F l^{3 \mathrm{D}}$ & $\begin{array}{l}\left\langle(-\mathbf{u} \cdot \nabla P)^{4}\right\rangle /\left\langle(-\mathbf{u} \cdot \nabla P)^{2}\right\rangle^{2} \\
\left\langle\left(\nu \mathbf{u} \cdot \nabla^{2} \mathbf{u}\right)^{4}\right\rangle /\left\langle\left(\nu \mathbf{u} \cdot \nabla^{2} \mathbf{u}\right)^{2}\right\rangle^{2}\end{array}$ & $\begin{array}{l}28.1 \\
30.0\end{array}$ & $\begin{array}{l}49.8 \\
51.2\end{array}$ & $\begin{array}{c}103.2 \\
56.3\end{array}$ \\
\hline$S k^{3 \mathrm{D}}$ & $\begin{array}{c}\left\langle p^{3}\right\rangle /\left\langle p^{2}\right\rangle^{3 / 2} \\
\left\langle(-\mathbf{u} \cdot \nabla P)^{3}\right\rangle /\left\langle(\mathbf{u} \cdot \nabla P)^{2}\right\rangle^{3 / 2} \\
\left\langle\left(\nu \mathbf{u} \cdot \nabla^{2} \mathbf{u}\right)^{3}\right\rangle /\left\langle\left(\nu \mathbf{u} \cdot \nabla^{2} \mathbf{u}\right)^{2}\right\rangle^{3 / 2}\end{array}$ & $\begin{array}{l}-0.53 \\
0.13 \\
-3.7\end{array}$ & $\begin{array}{l}-0.65 \\
0.15 \\
-4.3\end{array}$ & $\begin{array}{c}-0.67 \\
0.023 \\
-4.1\end{array}$ \\
\hline$M_{3}^{3 \mathrm{D}}$ & $\begin{array}{c}\left\langle(-\mathbf{u} \cdot \nabla P)^{3}\right\rangle /\left\langle p^{3}\right\rangle \\
\left\langle\left(\nu \mathbf{u} \cdot \nabla^{2} \mathbf{u}\right)^{3}\right\rangle /\left\langle p^{3}\right\rangle \\
3\left\langle(-\mathbf{u} \cdot \nabla P)^{2}\left(\nu \mathbf{u} \cdot \nabla^{2} \mathbf{u}\right)\right\rangle /\left\langle p^{3}\right\rangle \\
3\left\langle(-\mathbf{u} \cdot \nabla P)^{2}(\mathbf{u} \cdot \mathbf{f})\right\rangle /\left\langle p^{3}\right\rangle\end{array}$ & $\begin{array}{c}-0.24 \\
6.47 \times 10^{-2} \\
1.98 \\
*\end{array}$ & $\begin{array}{c}-0.19 \\
3.18 \times 10^{-2} \\
1.30 \\
*\end{array}$ & $\begin{array}{c}-0.034 \\
1.55 \times 10^{-2} \\
1.23 \\
-0.19 \\
\end{array}$ \\
\hline
\end{tabular}

of turbulent flows in 3D [22]. The observation of larger values of the flatness of $-(\mathbf{u} \cdot \nabla) P$ in $3 \mathrm{D}$ than in $2 \mathrm{D}$ (compare row $F l^{2 \mathrm{D}}$ of Table II and row $F l^{3 \mathrm{D}}$ of Table I) is in qualitative agreement with the stronger intermittency in $3 \mathrm{D}$ turbulent flows.

Whereas $-\mathbf{u} \cdot \nabla P$ clearly dominates the second and fourth moments of $p$, its influence on the third moment of $p$ is much more surprising. As shown in row $S k^{3 \mathrm{D}}$ of Table II, the skewness of $-\mathbf{u} \cdot \nabla P$ is slightly positive, which is in sharp contrast to the negative skewness of $p$. Although $\nu \mathbf{u} \cdot \nabla^{2} \mathbf{u}$ is negatively skewed, its small variance makes its contribution to $\left\langle p^{3}\right\rangle$ negligible (see row $M_{3}^{3 \mathrm{D}}$ of Table II). Thus, the skewness of $p$ cannot be explained by the third moment of any of the individual terms in Eq. (3) alone. In fact, the main contribution to the third moment of the power fluctuations comes from the cross-correlation of the pressure-gradient term with dissipation. Specifically, as shown in row $M_{3}^{3 \mathrm{D}}$ of Table II, the two dominant terms contributing to the third moment of power are

$$
\left\langle p^{3}\right\rangle \approx 3\left\langle(-\mathbf{u} \cdot \nabla P)^{2}\left(\nu \mathbf{u} \cdot \nabla^{2} \mathbf{u}\right)\right\rangle+3\left\langle(-\mathbf{u} \cdot \nabla P)^{2}(\mathbf{u} \cdot \mathbf{f})\right\rangle .
$$

The results of this section based on our investigation of the third moment of the power $p$ demonstrate that the role of pressure is very different in $2 \mathrm{D}$ and $3 \mathrm{D}$. While $\left\langle(-\mathbf{u} \cdot \nabla P)^{3}\right\rangle$ provides approximately $60 \%$ of $\left\langle p^{3}\right\rangle$ in $2 \mathrm{D}$, its contribution is very weak in $3 \mathrm{D}$, even with an opposite sign of that of $\left\langle p^{3}\right\rangle$. The small, slightly positive skewness of $-\mathbf{u} \cdot \nabla P$ in 3 implies that the strong action of the pressure force is more likely to contribute to large energy increase in $3 \mathrm{D}$ than in 2D. Section $\mathrm{V}$ sheds further light on this question.

\section{REDISTRIBUTION OF ENERGY BY PRESSURE FORCES}

The very different roles played by the pressure-gradient term $-\mathbf{u} \cdot \nabla P$ in $2 \mathrm{D}$ and in $3 \mathrm{D}$, together with the fact that $\langle\mathbf{u} \cdot \nabla P\rangle=0$, i.e., the pressure forces provide no net average kinetic energy change in statistically homogeneous and stationary turbulence, motivate us to ask how pressure redistributes energy among fluid particles. We surprisingly find that, while on average the pressure forces induce a very significant increase of the velocity of fast particles in 3D, this effect is almost completely absent in 2D.

This effect is demonstrated in Fig. 3, which shows the average pressure contribution to power on fluid particles conditioned upon kinetic energy, $\left\langle-\mathbf{u} \cdot \nabla P \mid \mathbf{u}^{2}\right\rangle$. In 2D, as shown in Fig. 3(a), $\left\langle-\mathbf{u} \cdot \nabla P \mid \mathbf{u}^{2}\right\rangle$ is very small, consistent with being 0 . This suggests that the redistribution of energy by pressure in $2 \mathrm{D}$ turbulent flows does not lead to any net effect (acceleration or deceleration) on either fast or slow particles. This lack of any net effect of pressure is the simplest way to satisfy Eq. (4). In sharp contrast, the surprising role of pressure in 3D turbulence is revealed by Fig. 3(b), which shows that the conditional mean $\left\langle-\mathbf{u} \cdot \nabla P \mid \mathbf{u}^{2}\right\rangle$ is negative for $\mathbf{u}^{2} /\left\langle\mathbf{u}^{2}\right\rangle \lesssim 2$ and positive for $\mathbf{u}^{2} /\left\langle\mathbf{u}^{2}\right\rangle \gtrsim 2$ (see also the recent results of Ref. [34]). That is, in 3D turbulence, pressure takes energy away from slow particles and gives it to fast particles. By itself the dynamics due to pressure forces would induce a runaway of the kinetic energy of some fluid elements, which has to be stopped eventually by viscous effects to prevent a singular behavior in the fluid. We note that the value of the conditional value $\left\langle-\mathbf{u} \cdot \nabla P \mid \mathbf{u}^{2}\right\rangle$ reaches values up to the standard deviation $\left\langle(\mathbf{u} \cdot \nabla P)^{2}\right\rangle^{1 / 2}$, which corresponds to $\approx 5$ times $\left\langle\left(\nu \mathbf{u} \cdot \nabla^{2} \mathbf{u}\right)^{2}\right\rangle^{1 / 2}$; see Fig. 2(b). 


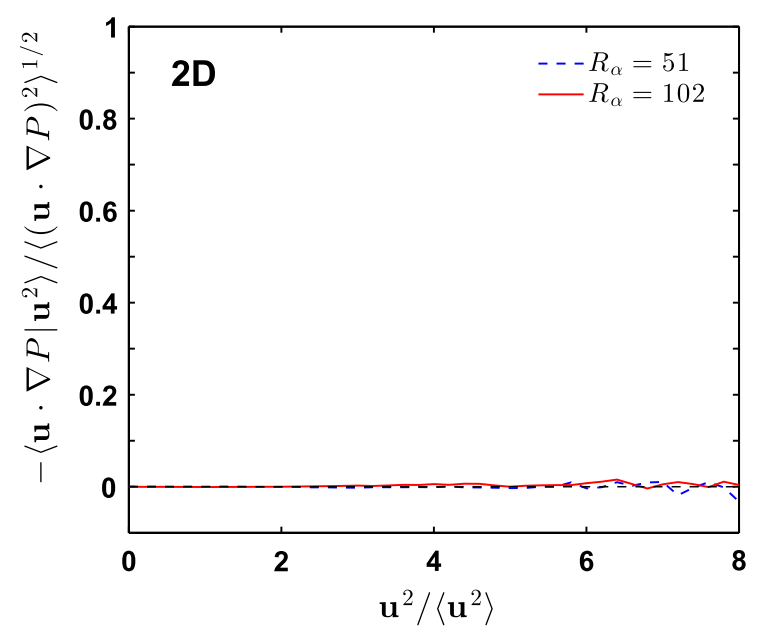

(a)

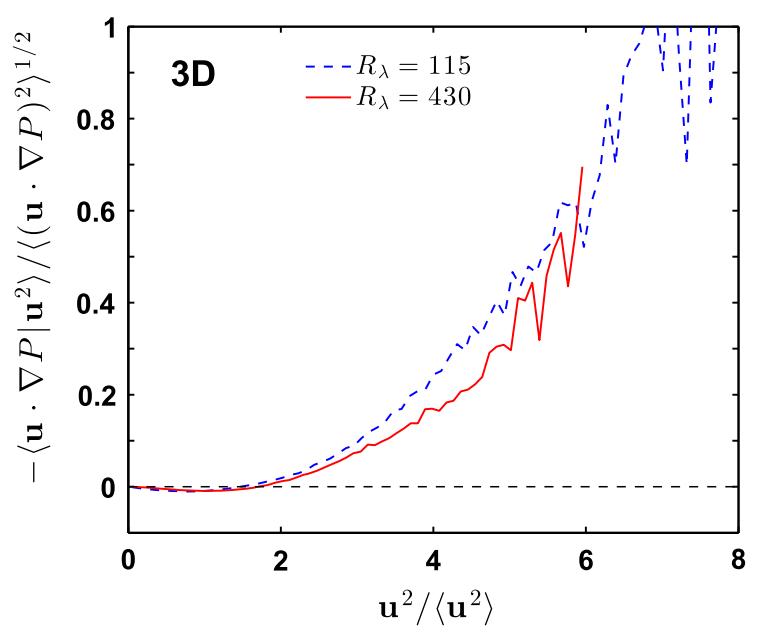

(b)

FIG. 3. The role of pressure in redistributing kinetic energy among fluid particles points to the dramatic difference between 2D and 3D turbulence. (a) The average of the pressure-gradient term conditioned on the kinetic energy of the fluid particle, $\left\langle-\mathbf{u} \cdot \nabla P \mid u^{2}\right\rangle$, in $2 \mathrm{D}$ turbulence (DNS data at $R_{\alpha}=51$ and $R_{\alpha}=102$ ). The average of $-\mathbf{u} \cdot \nabla P$, conditioned on $u^{2}$, is extremely small, and consistent with being 0 , implying that, on average, pressure does not redistribute energy among particles in $2 \mathrm{D}$ flows. (b) The same conditional average for 3D turbulence (DNS data at $R_{\lambda}=115$ and 430). Contrary to the 2D case, the conditional mean of the pressure term is negative for particles with small $u^{2}$, and becomes strongly positive for larger values of $u^{2}$, which implies that, on average, the pressure term in $3 \mathrm{D}$ turbulence takes energy from slow particles and gives to fast particles. This leads to a runaway effect and can be stopped only by viscous forces.

Thus, very large negative values of the $\nu \mathbf{u} \cdot \nabla^{2} \mathbf{u}$ are required to overcome a singular growth. While viscous dissipation is sufficient at the Reynolds numbers in our own simulations, whether the fluctuations of the dissipation remain large enough to counteract the effect of pressure at even higher Reynolds numbers, appears as a key question in maintaining a bounded kinetic energy of fluid particles. This problem

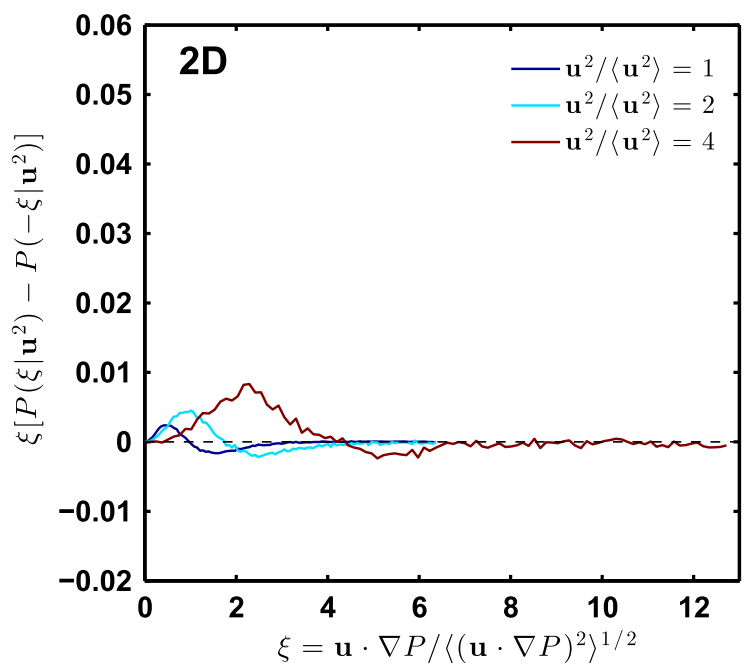

(a) calls not only for numerical studies at higher Reynolds numbers, but also for a deeper theoretical understanding. In particular, it is important to know whether the conditional mean $\left\langle-\mathbf{u} \cdot \nabla P \mid \mathbf{u}^{2}\right\rangle$ grows faster than $\mathbf{u}^{2}$, so that it can lead to blowup of particle energy in a finite time.

To further distinguish between the 2D and 3D cases, we investigate in detail the distribution of the normalized

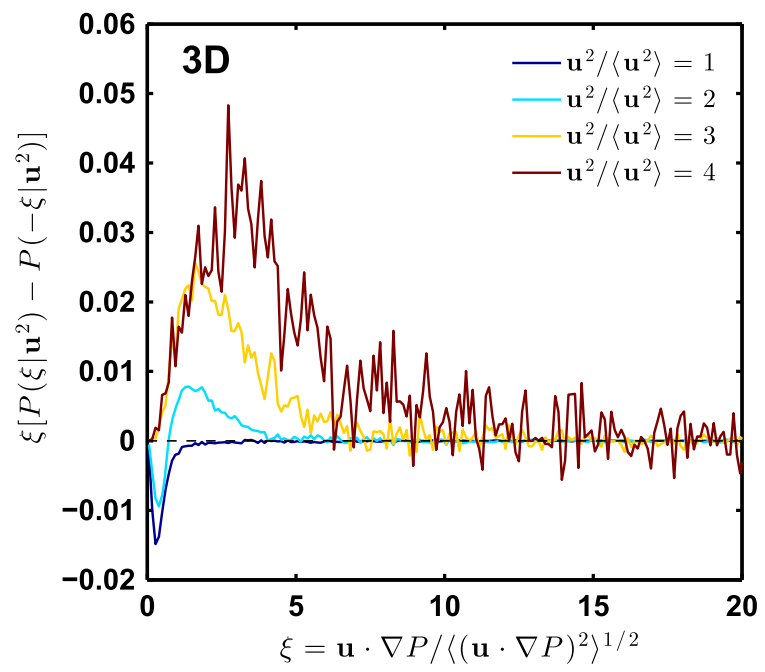

(b)

FIG. 4. The contribution of different values of $\xi \equiv-\mathbf{u} \cdot \nabla P /\left\langle(\mathbf{u} \cdot \nabla P)^{2}\right\rangle^{1 / 2}$ to the conditional average $\left\langle-\mathbf{u} \cdot \nabla P \mid \mathbf{u}^{2}\right\rangle$, at different values of $\mathbf{u}^{2}$. (a) $2 \mathrm{D}$ turbulence at $R_{\alpha}=102$ and (b) 3D turbulence at $R_{\lambda}=430$. The quantity shown is the difference between the PDF of positive and negative values of $\xi$, multiplied by $\xi$, so $\left\langle-\mathbf{u} \cdot \nabla P \mid \mathbf{u}^{2}\right\rangle$ is simply the integral of the curves shown in the figure. The quantity shown in $2 \mathrm{D}$ is generally much smaller than in $3 \mathrm{D}$. Also, at large values of $\mathbf{u}^{2}$ in $2 \mathrm{D}$, the very large values of $\xi$ tend to decelerate particles. In 3D, on the other hand, all values of $\xi$ contribute to transfer energy to fast particles. 
pressure contribution $\xi=-\mathbf{u} \cdot \nabla P /\left\langle(\mathbf{u} \cdot \nabla P)^{2}\right\rangle^{1 / 2}$. We note that the conditional averages of $\xi$ can be expressed as

$$
\left\langle\xi \mid \mathbf{u}^{2}\right\rangle=\int_{-\infty}^{\infty} \xi P\left(\xi \mid \mathbf{u}^{2}\right) d \xi=\int_{0}^{\infty} \xi\left[P\left(\xi \mid \mathbf{u}^{2}\right)-P\left(-\xi \mid \mathbf{u}^{2}\right)\right] d \xi,
$$

where $P\left(\xi \mid \mathbf{u}^{2}\right)$ is the conditional PDF of $\xi$. Figure 4 shows $\xi\left[P\left(\xi \mid \mathbf{u}^{2}\right)-P\left(-\xi \mid \mathbf{u}^{2}\right)\right]$, which, according to Eq. (10), represents the contribution of different values of $\xi$ to the conditional average since $\left\langle\xi \mid \mathbf{u}^{2}\right\rangle$ is simply the area under the curves shown. In general, the contribution of $\xi$ to the conditional average $\left\langle\xi \mid \mathbf{u}^{2}\right\rangle$ is 1 order of magnitude larger in 3D [Fig. 4(b)] than in 2D [Fig. 4(a)]. Also, at large values of $\mathbf{u}^{2}\left(\mathbf{u}^{2} \gtrsim 3\left\langle\mathbf{u}^{2}\right\rangle\right)$, the contributions of $\xi$ are always positive in $3 \mathrm{D}$, whereas in $2 \mathrm{D}$ the small values of $\xi$ contribute to accelerate fast particles, but the largest values of $\xi$ lead to a negative contribution (i.e., to a deceleration).

Our observation thus reveals a strong difference between $3 \mathrm{D}$ and $2 \mathrm{D}$ turbulent flows. In 3D, the results shown in Fig. 3(b) suggest an unexpected mechanism, which could lead to an increase of the kinetic energy of particles by the only action of the pressure forces. Such a mechanism is particularly interesting for a deeper understanding of the physics of turbulent flows.

\section{SUMMARY AND DISCUSSION}

We investigate the rate of change of kinetic energy, or the instantaneous power $p=\mathbf{u} \cdot \mathbf{a}$, of individual fluid particles in homogeneous turbulent flows in both $2 \mathrm{D}$ and $3 \mathrm{D}$. To this end, we use the decomposition of the power $p$, based on Eq. (3), which amounts to a budget of the work done by the forces acting on individual fluid particles, resulting from the pressure gradient, dissipation (due to viscosity and friction), and external forcing in the Navier-Stokes equation.

We find that while the net mean of the pressure contribution to power vanishes, $\langle-\mathbf{u} \cdot \nabla P\rangle=0$, it provides the main contribution to the fluctuations of the instantaneous power, both in $2 \mathrm{D}$ and in $3 \mathrm{D}$. This means that the pressure term merely redistributes energy among fluid elements without causing net overall energy change. The conditional average $\left\langle-\mathbf{u} \cdot \nabla P \mid \mathbf{u}^{2}\right\rangle$ reveals striking differences in the role of pressure forces in $2 \mathrm{D}$ and $3 \mathrm{D}$. Whereas in 2D pressure forces do not result in an average transfer of energy towards slow or fast particles, they tend to accelerate the fastest particles in 3D.

At the moderate Reynolds numbers investigated here $\left(R_{\lambda} \leq 430\right)$, the accelerating effect of the pressure forces is compensated by the stabilizing effect of the dissipative (viscous) forces. To draw conclusions on whether at much higher Reynolds numbers pressure forces can prevail over the action of dissipation, so as to lead to an unbounded growth of velocity, requires, in addition to data at higher resolution, a better understanding of the action of pressure forces. The potential insight gained by further studying the mechanism, based on pressure forces, of amplification of the velocity, and thus, of the velocity gradient, is likely to provide new information about the structure of three-dimensional turbulent flows. In particular, the possibility that pressure could induce a runaway of the velocity may be related to the accelerated nature of the turbulence energy cascade in three dimensions [35]. In any event, the observation that pressure forces tend, on average, to accelerate fast particles provides a strong motivation to better analyze and understand the subtle, nonlocal effect of pressure forces, which remain a major challenge. In physical terms, an understanding of turbulence is needed that goes well beyond the analysis in terms of elementary flow structures $[21,33]$.

From the mathematical point of view, the existence of a mechanism leading to an acceleration of very fast particles in 3D is appealing, as it could possibly lead to an unbounded growth of velocity, which is known to be a necessary condition for the appearance of singularities of the Navier-Stokes equations [19]. Providing bounds on this role of pressure could lead to important insight on the corresponding Clay Institute millennium problem, for which, as explicitly stated in Ref. [20], "some deep, new ideas are needed." We are very much aware that our work may merely serve as a proposal and only detailed mathematical analysis will lead to firm conclusions on the fundamental question of regularity of the Navier-Stokes equations.

\section{ACKNOWLEDGMENTS}

We acknowledge informative discussions with Michael Wilczek. Financial support from the Max Planck Society is gratefully appreciated. A. P. has been supported by the grant TEC 2 from the "Agence Nationale pour la Recherche" (Grant No. ANR-12-BS09-0011-02), and by fellowship from the Alexander von Humboldt foundation. The work of G. F. has been supported by the grant of the Minerva Foundation, with funding from the German Ministry for Education and Research. This work is supported by EuHIT- European Highperformance Infrastructure in Turbulence, which is funded by the European Commission Framework Program 7 (Grant No. 312778). Numerical simulations were performed at the PSMN computing center at the Ecole Normale Supérieure de Lyon and on the "Turbofarm" cluster at the University of Torino.

\section{APPENDIX A: HIGHER MOMENTS OF POWER STATISTICS IN 2D TURBULENCE}

Based on Fig. 5, here, we discuss the statistical properties of the contributions of the pressure gradient, of the dissipative terms, and of the forcing to the power acting on a fluid particle in 2D flows.

Figures $5(\mathrm{a})-5(\mathrm{c})$ show the distribution of $-(\mathbf{u} \cdot \nabla P)$, $\nu \mathbf{u} \cdot \nabla^{2} \mathbf{u}$, and $-\alpha \mathbf{u}^{2}$, respectively, all normalized by their standard deviations. The quantity whose distribution exhibits the strongest variation as a function of $R_{\alpha}$ is the 


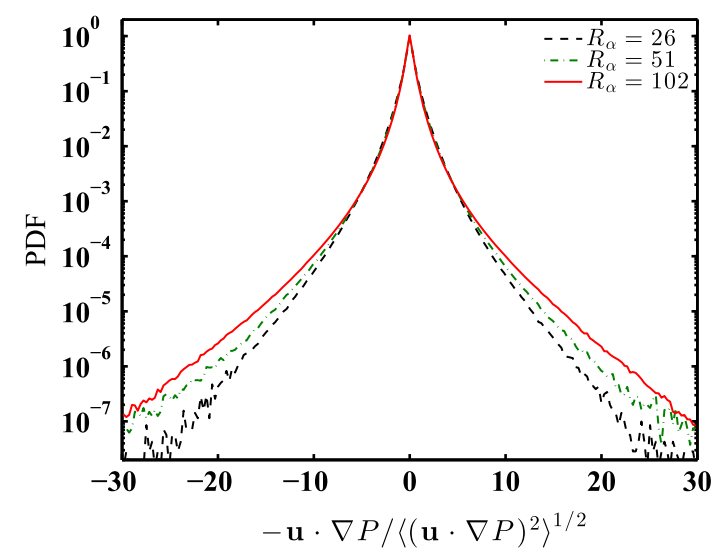

(a)

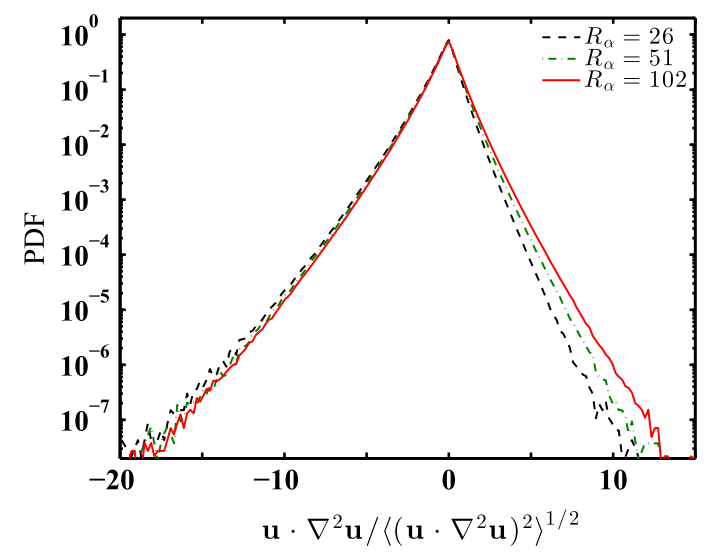

(b)

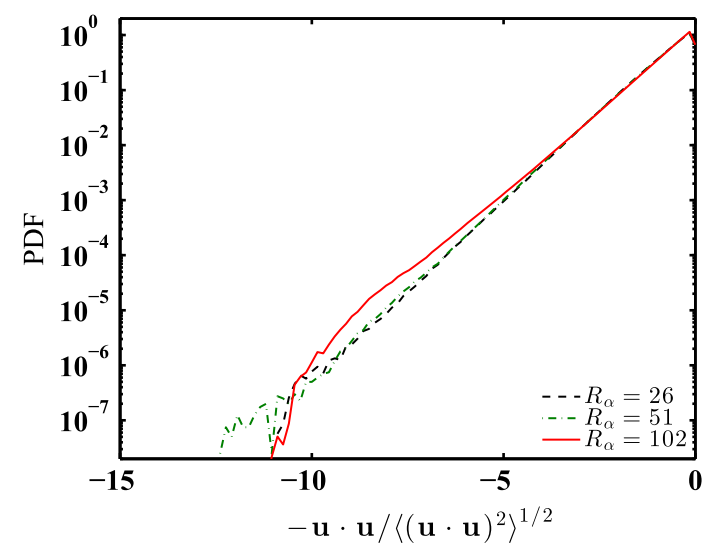

(c)

FIG. 5. PDFs of (a) the pressure term $-\mathbf{u} \cdot \nabla P$, (b) the viscous term $\nu \mathbf{u} \cdot \nabla^{2} \mathbf{u}$, and (c) the friction term $-\alpha \mathbf{u}^{2}$, normalized by their standard deviations, in 2D turbulence. The Reynolds numbers of the flows shown are $R_{\alpha}=26,51$, and 102. The PDFs of $-\mathbf{u} \cdot \nabla P$ are slightly asymmetric, while the skewness of $\nu \mathbf{u} \cdot \nabla^{2} \mathbf{u}$ is much larger in magnitude, from $\approx-2.0$ at $R_{\alpha}=26$ to $\approx-1.2$ at $R_{\alpha}=102$. The exponential tails seen in the PDFs of $-\alpha \mathbf{u}^{2}$ reflect the (approximately) Gaussian distribution of $\mathbf{u}$. Both the flatness $(\approx 6)$ and the skewness $(\approx-2)$ of the friction term are approximately constant over the range of Reynolds numbers considered. contribution from the pressure gradient, $-\mathbf{u} \cdot \nabla P$. The distribution shown in Fig. 5(a) exhibits heavy tails, more so as the Reynolds number $R_{\alpha}$ becomes larger. This tendency is reflected in the dependence of the flatness on $R_{\alpha}$, shown in row $F l^{2 \mathrm{D}}$ in Table I. Although it has been observed many times, based on the study of Eulerian structure functions, that turbulence in 2D is not intermittent, we find, unexpectedly, that the fluctuations of the quantity $-\mathbf{u} \cdot \nabla P$ become stronger as the Reynolds number increases. This surprising effect clearly deserves further investigation.

The PDFs of the viscous dissipation term, $\nu \mathbf{u} \cdot \nabla^{2} \mathbf{u}$, are clearly asymmetric, with more pronounced negative (corresponding to dissipation events) than positive tails. The negative tails are approximately independent of $R_{\alpha}$. In contrast, the positive tails, corresponding to situations where viscosity induces kinetic energy gains, become broader when $R_{\alpha}$ increases. The observed changes in the distribution do not lead to a very significant change in the flatness; see row $F l^{2 \mathrm{D}}$ of Table I. The skewness of the viscous dissipation term, however, decreases by approximately $40 \%$ in magnitude, from $\approx-2.0$ at $R_{\alpha}=26$ to $\approx-1.2$ at $R_{\alpha}=102$.

The frictional dissipation $-\alpha \mathbf{u}^{2}$, whose PDFs are shown in Fig. 5(c), is by definition a negative quantity. The PDFs exhibit tails that are very close to being exponential, which can be qualitatively understood from the Gaussian character of the velocity fluctuations. The exponential tails are essentially independent of the Reynolds number, as seen from the nearly constant skewness $(\approx-2)$ and flatness $(\approx 6)$, both independent of $R_{\alpha}$ (see rows $S k^{2 \mathrm{D}}$ and $F l^{2 \mathrm{D}}$ of Table I.

The direct contributions to the third moment of power $p$, due to the dissipation terms $-\alpha \mathbf{u}^{2}$ and $\nu \mathbf{u} \cdot \nabla^{2} \mathbf{u}$, are very small (see row $M_{3}^{2 \mathrm{D}}$ of Table I). These quantities have very asymmetric distributions, but their variances are much smaller than that of the distribution of power, as seen from $M_{2}^{2 D}$ in Table I and Fig. 2(a).

\section{APPENDIX B: HIGHER MOMENTS OF POWER STATISTICS IN 3D TURBULENCE}

Here, we provide more detailed information about the contributions of the pressure gradient, the viscous dissipation, and forcing to the power acting on a fluid particle in 3D flows.

Figure 6 shows the PDFs of $-\mathbf{u} \cdot \nabla P$ and the viscous dissipation term $\nu \mathbf{u} \cdot \nabla^{2} \mathbf{u}$ in 3D. As for the 2D cases, the two quantities exhibit heavy probability tails, which become wider as the Reynolds number $R_{\lambda}$ increases. The growth of these tails when $R_{\lambda}$ increases is consistent with the information on the flatness, listed in row $F l^{3 \mathrm{D}}$ in Table II. It is a manifestation of intermittency, a characteristic property of turbulent flows [22]. As indicated by the flatness values shown in row $F l^{3 \mathrm{D}}$ in Table II, the extension of the heavy tails is more pronounced for the pressure term, $-\mathbf{u} \cdot \nabla P$, than for the viscous term, $\nu \mathbf{u} \cdot \nabla^{2} \mathbf{u}$. 


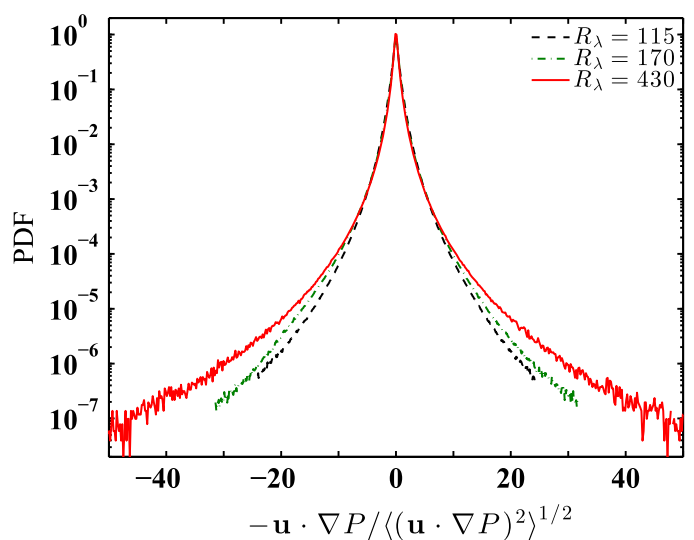

(a)

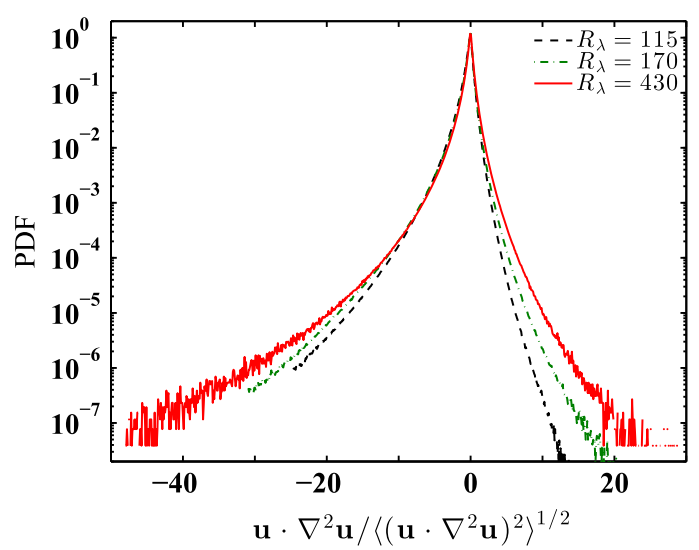

(b)

FIG. 6. PDFs of (a) $-\mathbf{u} \cdot \nabla P$ and (b) $\nu \mathbf{u} \cdot \nabla^{2} \mathbf{u}$ normalized by their standard deviations in 3D flows. The Reynolds numbers are $R_{\lambda}=115,170$, and 430 . The PDFs of both quantities exhibit heavy tails. The asymmetry of $-\mathbf{u} \cdot \nabla P$ is very weak (the magnitudes of the skewness of the distributions are smaller than $\sim 0.15$ ). In contrast, the PDFs of $\nu \mathbf{u} \cdot \nabla^{2} \mathbf{u}$ are highly asymmetric, with skewness of approximately -4 .

Although the pressure-gradient term $-\mathbf{u} \cdot \nabla P$ is the dominant contribution to the variance of $p$, this term alone does not provide the main contribution to the third moment $\left\langle p^{3}\right\rangle$. Figure 6(a) shows that the PDF of $-\mathbf{u} \cdot \nabla P$ is nearly symmetrical. In fact, the skewness of $-\mathbf{u} \cdot \nabla P$ is even found to be slightly positive, although it remains to be seen whether this property holds at even higher Reynolds numbers. At the highest value of $R_{\lambda}$ considered here $\left(R_{\lambda}=430\right)$, the skewness $\left\langle(-\mathbf{u} \cdot \nabla P)^{3}\right\rangle /\left\langle(-\mathbf{u} \cdot \nabla P)^{2}\right\rangle^{3 / 2}$ is very small, $\approx 0.023$. This is to be contrasted with the skewness of power $\left\langle p^{3}\right\rangle /\left\langle p^{2}\right\rangle^{3 / 2}$, which is -0.67 at $R_{\lambda}=430$.

Figure 6(b) shows that the asymmetry of the distribution of $\nu \mathbf{u} \cdot \nabla^{2} \mathbf{u}$ is very strong, and results in a skewness of approximately -4 , nearly independent of the Reynolds number (see row $S k^{3 \mathrm{D}}$ of Table II). The third moment of $\nu \mathbf{u} \cdot \nabla^{2} \mathbf{u}$, however, does not contribute significantly to the third moment of $p$, because of the small variance of $\nu \mathbf{u} \cdot \nabla^{2} \mathbf{u}$ (see row $M_{2}^{3 \mathrm{D}}$ of Table II). The third moment of $\mathbf{u} \cdot \mathbf{f}$ is even weaker.

Thus, the asymmetry of the PDF of power $p$ cannot be explained by the third moment of any of the individual terms in Eq. (3). As stated in Eq. (9) (see also $M_{3}^{3 \mathrm{D}}$ in Table II), the main contribution to the third moment of the power fluctuations comes from the cross-correlation of the pressure with dissipation.

[1] G. K. Batchelor, Pressure Fluctuations in Isotropic Turbulence, Proc. Cambridge Philos. Soc. 47, 359 (1951).

[2] A. M. Obukhov and A. M. Yaglom, The Microstructure of Turbulent Flow, Prikl. Mat. Mekh. 15, 3 (1951); [NACA TM 1350 (1953)].

[3] R. J. Hill and J. M. Wilczak, Pressure Structure Functions and Spectra for Locally Isotropic Turbulence, J. Fluid Mech. 296, 247 (1995).

[4] R. J. Hill and S. T. Thoroddsen, Experimental Evaluation of Acceleration Correlations for Locally Isotropic Turbulence, Phys. Rev. E 55, 1600 (1997).

[5] A. La Porta, G. A. Voth, A. M. Crawford, J. Alexander, and E. Bodenschatz, Fluid Particle Accelerations in Fully Developed Turbulence, Nature (London) 409, 1017 (2001).

[6] G. A. Voth, A. La Porta, A. M. Crawford, J. Alexander, and E. Bodenschatz, Measurement of Particle Accelerations in Fully Developed Turbulence, J. Fluid Mech. 469, 121 (2002).

[7] H. Xu, N. T. Ouellette, D. Vincenzi, and E. Bodenschatz, Acceleration Correlations and Pressure Structure Functions in High-Reynolds Number Turbulence, Phys. Rev. Lett. 99, 204501 (2007).

[8] P. Vedula and P. K. Yeung, Similarity Scaling of Acceleration and Pressure Statistics in Numerical Simulations of Isotroic Turbulence, Phys. Fluids 11, 1208 (1999).

[9] T. Gotoh and D. Fukayama, Pressure Spectrum in Homogeneous Turbulence, Phys. Rev. Lett. 86, 3775 (2001).

[10] Y. Tsuji and T. Ishihara, Similarity Scaling of Pressure Fluctuation in Turbulence, Phys. Rev. E 68, 026309 (2003).

[11] R. J. Hill, Equations Relating Structure Functions of All Orders, J. Fluid Mech. 434, 379 (2001).

[12] T. Gotoh and T. Nakano, Role of Pressure in Turbulence, J. Stat. Phys. 113, 855 (2003).

[13] G. Falkovich, I. Fouxon, and Y. Oz, New Relations for Correlation Functions in Navier-Stokes Turbulence, J. Fluid Mech. 644, 465 (2010).

[14] H. Xu, A. Pumir, G. Falkovich, E. Bodenschatz, M. Shats, H. Xia, N. Francois, and G. Boffetta, Power Fluctuations and Irreversibility in Turbulence, arXiv:1310.5006.

[15] H. Xu, A. Pumir, G. Falkovich, E. Bodenschatz, M. Shats, H. Xia, N. Francois, and G. Boffetta, Flight-Crash Events in Turbulence, Proc. Natl. Acad. Sci. U.S.A. 111, 7558 (2014).

[16] T. von Kármán and L. Howarth, On the Statistical Theory of Isotropic Turbulence, Proc. R. Soc. A 164, 192 (1938).

[17] A. S. Monin and A. M. Yaglom, Statistical Fluid Mechanics: Mechanics of Turbulence (MIT, Cambridge, MA, 1975), Vol. 2. 
[18] L. D. Landau and E. M. Lifshitz, Fluid Mechanics (Butterworth-Heinemann, Oxford, UK, 1987).

[19] J. Leray, Sur le Mouvement d'un Fluide Visqueux Emplissant l'Espace, Acta Math. 63, 193 (1934).

[20] C. L. Fefferman, The Millennium Prize Problems (Clay Mathematics Institute, Cambridge, MA, 2006), pp. 57-67.

[21] T. Tao, Finite Time Blowup for an Averaged ThreeDimensional Navier-Stokes Equation, arXiv:1402.0290v2.

[22] U. Frisch, Turbulence: The Legacy of A. N. Kolmogorov (Cambridge University Press, Cambridge, England, 1995).

[23] A. Pumir, A Numerical Study of Pressure Fluctuations in Three-Dimensional, Incompressible, Homogeneous, Isotropic Turbulence, Phys. Fluids 6, 2071 (1994).

[24] Y. Li, E. Perlman, M. Wan, Y. Yang, C. Meneveau, R. Burns, S. Chen, A. Szalay, and G. L. Eyink, A Public Turbulence Database Cluster and Applications to Study Lagrangian Evolution of Velocity Increments in Turbulence, J. Turbul. 9, 31 (2008).

[25] R. H. Kraichnan, Inertial Ranges in Two-Dimensional Turbulence, Phys. Fluids 10, 1417 (1967).

[26] G. Boffetta and R. E. Ecke, Two-Dimensional Turbulence, Annu. Rev. Fluid Mech. 44, 427 (2012).

[27] G. Boffetta and S. Musacchio, Evidence for the Double Cascade Scenario in Two-Dimensional Turbulence, Phys. Rev. E 82, 016307 (2010).

[28] M. M. Bandi and C. Connaughton, Craigs XY Distribution and the Statistics of Lagrangian Power in Two-Dimensional Turbulence, Phys. Rev. E 77, 036318 (2008).
[29] N. Francois, H. Xia, H. Punzmann, and M. Shats, Inverse Energy Cascade and Emergence of Large Coherent Vortices in Turbulence Driven by Faraday Waves, Phys. Rev. Lett. 110, 194501 (2013).

[30] A. von Kameke, F. Huhn, G. Fernandez-Garcia, A. P. Munuzuri, and V. Perez-Munuzuri, Double Cascade Turbulence and Richardson Dispersion in a Horizontal Fluid Flow Induced by Faraday Waves, Phys. Rev. Lett. 107, 074502 (2011).

[31] Different properties of the power of the forcing term acting on a tracer particle, $\mathbf{u} \cdot \mathbf{f}$, may be observed if other forcing schemes are used. In particular, if the forcing is Gaussian and has a long correlation time, the PDF of the power term $\mathbf{u} \cdot \mathbf{f}$ displays skewed tails, which could be understood as the product of two correlated Gaussian random variables [28].

[32] P. K. Yeung, S. B. Pope, A. G. Lamorgese, and D. A. Donzis, Acceleration and Dissipation Statistics of Numerically Simulated Isotropic Turbulence, Phys. Fluids 18, 065103 (2006).

[33] T. Ishihara, Y. Kaneda, M. Yokokawa, K. Itakura, and A. Uno, Small-Scale Statistics in High-Resolution Direct Numerical Simulation of Turbulence: Reynolds Number Dependence of One-Point Velocity Gradient Statistics, J. Fluid Mech. 592, 335 (2007).

[34] M. Wilczek, A. Daitche, and R. Friedrich, On the Velocity Distribution in Homogeneous Isotropic Turbulence: Correlations and Deviations from Gaussianity, J. Fluid Mech. 676, 191 (2011).

[35] G. Falkovich and K. Sreenivasan, Lessons from Hydrodynamic Turbulence, Phys. Today 59, No. 4, 43 (2006). 